\title{
Dissection of QTL effects for root traits using a chromosome arm-specific mapping population in bread wheat
}

\author{
Sundrish Sharma $\cdot$ Shizhong Xu $\cdot$ Bahman Ehdaie • \\ Aaron Hoops • Timothy J. Close $\cdot$ Adam J. Lukaszewski • \\ J. Giles Waines
}

Received: 25 February 2010/ Accepted: 22 October 2010/Published online: 11 December 2010

(C) The Author(s) 2010. This article is published with open access at Springerlink.com

\begin{abstract}
A high-resolution chromosome arm-specific mapping population was used in an attempt to locate/detect gene(s)/QTL for different root traits on the short arm of rye chromosome 1 (1RS) in bread wheat. This population consisted of induced homoeologous recombinants of 1RS with 1BS, each originating from a different crossover event and distinct from all other recombinants in the proportions of rye and wheat chromatin present. It provides a simple and powerful approach to detect even small QTL effects using fewer progeny. A promising empirical Bayes method was applied to estimate additive and epistatic effects for all possible marker pairs simultaneously in a single model. This method has an advantage for QTL analysis in minimizing the error variance and detecting interaction effects between loci with no main effect. A total of 15 QTL effects, 6 additive and 9 epistatic, were detected for different traits of root length and root weight in 1RS wheat. Epistatic interactions were further partitioned into inter-genomic (wheat and rye alleles) and intra-genomic (rye-rye or wheat-wheat alleles) interactions affecting various root traits. Four common regions were identified involving all the QTL for root traits. Two regions carried QTL for almost all the root traits and were responsible for all the epistatic interactions. Evidence for inter-genomic interactions is provided. Comparison of mean values supported the QTL detection.
\end{abstract}

Communicated by J. Snape.

S. Sharma $\cdot$ S. Xu · B. Ehdaie · A. Hoops .

T. J. Close · A. J. Lukaszewski · J. G. Waines ( $\square)$

Department of Botany and Plant Sciences,

University of California, Riverside, CA 92521-0124, USA

e-mail: giles.waines@ucr.edu

Present Address:

S. Sharma

Syngenta Seeds, Inc., Naples, FL 34114, USA

\section{Introduction}

Roots, the hidden half of a plant, are important for numerous functions including water and nutrient uptake that make it difficult to overlook their importance to plant productivity (MacMillan et al. 2006). It is an irony that this organ has inspired so few plant scientists to work relative to the number who work on above-ground plant parts. The limited research effort in improvement of roots may be because of the difficulty in observing, measuring, and manipulating them (Shen et al. 2001).

Rye (Secale cereale L., $2 n=2 x=14$, genome formula $\mathrm{RR}$ ) is well known for its abiotic stress tolerance (Hackauf et al. 2009) and resistance against diseases and pests (Zeller and Hsam 1984). Very likely, those resistances facilitated the selection and establishment of a spontaneous centric rye-wheat translocation 1RS.1BL in place of chromosome $1 \mathrm{~B}$ of bread wheat (Triticum aestivum L., $2 n=6 x=42$, genome formula BBAADD) (Mettin et al. 1973). The translocation spread throughout the world even when these resistance genes were overcome and eventually made it into hundreds of released cultivars (Braun et al. 1998). It was realized that the translocation increased grain yield even in the absence of pathogens (Villareal et al. 1991), and this yield gain was partly attributed to a substantially increased root biomass (Ehdaie et al. 2003; Ehdaie and Waines 2008). A larger root system increases uptake of water and nutrients from the soil (Ehdaie et al. 2003).

Cereal roots have two main classes, seminal roots and nodal roots (Esau 1965). Seminal roots originate from the germinating embryonic hypocotyls, and nodal roots emerge from the coleoptile nodes at the base of the apical culm (Manske and Vlek 2002). Weaver (1926) compared the root systems of rye and bread wheat, and reported rye had 
longer seminal roots. The genetic control of root characteristics is poorly understood as the growth pattern changes greatly depending on the environment and it is obscured from direct observation. Root traits are believed to be complex and controlled by many genes, each with a small genetic effect. Genetic loci controlling such traits are called quantitative trait loci (QTL). With the advent of molecular markers, it has become possible to estimate the genome location and size of QTL, including those for root characters. Research has recently been undertaken to map root QTL in rice (Oryza sativa L.) (Champoux et al. 1995; Price and Tomos 1997; Zheng et al. 2000), maize (Zea mays L.) (Lebreton et al. 1995), common bean (Phaseolus vulgaris L.) (Ochoa et al. 2006), and Arabidopsis (Gerald et al. 2006). In wheat, many QTL have been identified for aboveground traits of agronomic importance (Spielmeyer et al. 2007; Maccaferri et al. 2008; Zhang et al. 2008) but no information on root genes or QTL has been reported. Disregarding root pathogens, the most recent wheat gene catalog contains not a single reference to roots (http:// wheat.pw.usda.gov/GG2/Triticum/wgc/2008/; McIntosh et al. 2003).

Most quantitative traits are determined by many interacting loci with small genetic effects that are modified by environmental factors (Falconer and Mackay 1996). Interaction of these alleles at different loci is called epistasis (Fisher 1918; Holland 2001). Epistasis is now considered an important source of genetic variation with some components, especially Additive $\times$ Additive receiving more attention (Goodnight 2000; Jannink 2003) due to their heritable nature (Ehdaie and Cress 1973). Efficient methods have been developed to map QTL with additive (main) effects (Lander and Botstein 1989; Xu 2003a; Zhang et al. 2005), but mapping QTL with epistatic effects is still at an early stage. There were efforts to detect epistasis using Bayesian models (Yi et al. 2003, 2006), but they were unable to guarantee detection of all such effects. The Bayesian approach uses a given prior distribution (a prior estimate of an unobserved parameter) and Markov chain Monte Carlo (MCMC) sampling to infer posterior distribution conditional on the data (observable) (Wu et al. 2007). Recently, Xu (2007) developed an empirical Bayes method (E-BAYES) that requires no MCMC samplings, yet still estimates the variance parameters for the priors of the regression coefficients. Simultaneous estimation of individual additive (main) effects of all markers and their epistatic effects in all combinations make this approach useful to study gene interactions where many may go undetected.

Recent studies have shown the efficiency of chromosome-specific mapping populations over traditional crosses in detecting a given effect with fewer progenies (Singer et al. 2004). The power of such a population, in a statistical sense, has been demonstrated in animal studies such as mice (Mus musculus) (Nadeau et al. 2000; Belknap 2003; Singer et al. 2004). In plants, stepped aligned recombinant inbred strains (STAIRS) were generated in Arabidopsis, using chromosome substitution strains (Koumproglou et al. 2002), but there is no detailed report until now on using such a mapping population for analysis of complex traits. It is another irony that chromosome substitution lines have been incorporated in wheat genetics and breeding programs since the 1950s (Sears 1953; Law et al. 1987), but it is only now when they were re-invented in Arabidopsis that they are being appreciated for generating chromosome-specific mapping populations to study quantitative traits. This article presents a first ever attempt to characterize QTL effects for wheat root traits, using the E-BAYES method in combination with a chromosome-specific mapping population. Here, we report the detection of additive and epistatic effects and also further dissection of gene interaction effects into inter-genomic and intra-genomic epistatic effects.

\section{Materials and methods}

\section{Mapping population}

The root study was done on 29 recombinant lines, each having a different recombination breakpoint. These 29 lines were selected from a population of 68 1RS-1BS recombinants used earlier to generate the 1RS-1BS integrated map (Sharma et al. 2009), to represent all map intervals. "Pavon 76" (spring wheat from CIMMYT, Mexico) and Pavon 1RS.1BL were used as parents to generate the mapping population; recombination of 1RS1BS was induced by the absence of the Phl locus (Lukaszewski 2000). The integrated map consisted of 20 polymorphic physical and molecular markers in 15 intervals spanning 35-40\% of the physical length of the chromosome arm, with average spacing of ca. $2.5 \mathrm{cM}$ (Sharma et al. 2009). Given that each translocation is a product of an independent and single crossover event, the total map length must equal $50 \mathrm{cM}$, and the 68 1RS-1BS recombination breakpoints produced the average map resolution of $0.7 \mathrm{cM}$. The recombinants were divided into two groups on the basis of the configuration: the rye segment in a terminal position $(1 \mathrm{~B}+$ lines $)$ or rye segment in a proximal position (T-lines).

\section{Phenotyping}

In addition to the 29 recombinant lines viz., $1 \mathrm{~B}+1,1 \mathrm{~B}+2$, $1 \mathrm{~B}+5,1 \mathrm{~B}+6,1 \mathrm{~B}+12,1 \mathrm{~B}+14,1 \mathrm{~B}+19,1 \mathrm{~B}+25,1 \mathrm{~B}+32$, $1 \mathrm{~B}+35,1 \mathrm{~B}+38,1 \mathrm{~B}+40,1 \mathrm{~B}+42,1 \mathrm{~B}+44,1 \mathrm{~B}+53, \mathrm{~T}-1$, 
T-9, T-14, T-15, T-16, T-18, T-26, T-27, T-32, T-33, T-34, T-47, T-50, and T-51, the experiment also included Pavon 76, Pavon 1RS.1BL, and Pavon Dt. 1BL as checks, for the total of 32 lines. All materials were grown under natural photoperiod in a temperature-controlled glasshouse $\left(20-30^{\circ} \mathrm{C}\right.$ and $50-90 \%$ relative humidity) in a randomized complete block design with four replications during Spring 2007, Fall 2007, Winter 2008, and Fall 2008. Plants were grown in sand culture in $80 \mathrm{~cm}$ long and $10 \mathrm{~cm}$ wide PVC tubes, with polyethylene tubular inner sleeves and regularly watered with half-strength Hoagland's solution to ensure adequate nutrient supply. Plants were harvested 45 days after germination at the mid-late tillering stage, and roots were washed free of sand by the floatation technique (Böhm 1979). Various plant characters were measured. The shoot characters measured were; the longest leaf length $(\mathrm{LL})(\mathrm{cm})$, maximum width of the longest leaf $(\mathrm{LW})(\mathrm{cm})$, leaf area $(\mathrm{LA})\left(\mathrm{cm}^{2}\right)$, plant height $(\mathrm{PH})(\mathrm{cm})$, number of tillers per plant (NT), dry shoot biomass (SB) (g), and the root characters were number of roots greater than $30 \mathrm{~cm}$ (NR), longest root length (LRL) $(\mathrm{cm})$, total length of roots greater than $30 \mathrm{~cm}$ (TRL) $(\mathrm{cm})$, shallow root weight (depth $<30 \mathrm{~cm})$ (SRW) (g), deep root weight (depth $>$ $30 \mathrm{~cm})(\mathrm{DRW})(\mathrm{g})$, total root weight (TRW) (g), and root biomass to shoot biomass ratio $(\mathrm{R} / \mathrm{S})$.

The genotype of each marker was coded as +1 for the wheat allele and -1 for the rye allele. The overall mean phenotypic value of each line across the environments was taken as the input phenotype for that line representing the genotypic value of that line. All QTL detected would represent those showing consistent effects across environments.

\section{Statistical analysis}

The empirical Bayes method was used in the data analysis (Xu 2007). The linear model to describe the vector of phenotypic values for a trait is:

$\mathbf{y}=1 \mu+\sum_{l=1}^{m} \mathbf{G}_{l} \gamma_{l}+\sum_{l^{\prime}>l}^{m}\left(\mathbf{G}_{l} \times \mathbf{G}_{l^{\prime}}\right) \gamma_{l^{\prime}}+\varepsilon$,

where $\mathbf{y}$ is an $n \times 1$ vector, $\mu$ is the population mean, $\mathbf{G}_{l}$ is genotype indicator variable for a given locus $l, \gamma_{l}$ is the additive effect for locus $l, \gamma_{l l^{\prime}}$ is epistatic effect between loci $l$ and $l^{\prime}$, and $\varepsilon$ is the residual error. The notation $\mathbf{G}_{l} \times \mathbf{G}_{l^{\prime}}$ represents the direct product of vectors $\mathbf{G}_{l}$ and $\mathbf{G}_{l^{\prime}}$. Excluding $\mu$, the total number of QTL effects for $m=15$ markers is $p=m(m+1) / 2=120$, including $m=15$ main effects and $m(m-1) / 2=105$ pair-wise epistatic effects. We now use $j$ to index the $j$ th genetic effect (including additive and pair-wise epistatic effects) for $j=1, \ldots, p$. We can rewrite model (1) as $\mathbf{y}=1 \mu+\sum_{j=1}^{p} \mathrm{X}_{j} \beta_{j}+\varepsilon$.

Comparing model (2) to model (1), we can see that $\mathbf{X}_{j}=\mathbf{G}_{l}$ and $\beta_{j}=\gamma_{l}$ if the $j$ th effect is a main effect, and $\mathbf{X}_{j}=\mathbf{G}_{l} \times \mathbf{G}_{l^{\prime}}$ and $\beta_{j}=\gamma_{l l^{\prime}}$ if the $j$ th effect is an epistatic effect. Therefore, model (2) is a general model for both the main and the epistatic effects. As far as the method of estimating genetic effects is concerned, distinction between a main effect and an epistatic effect is unnecessary (Xu and Jia 2007).

QTL effects were estimated by the two-step approach of $\mathrm{Xu}$ (2007). First, variance components were estimated by a typical random model variance-component analysis using maximum-likelihood method (Hartley and Rao 1967) and second, estimation of QTL effect by best linear unbiased prediction (BLUP) given the estimated variance components (Robinson 1991; Xu and Jia 2007). This analysis provided BLUP estimates of QTL effects $\beta_{j}$ and that value was squared to calculate the genetic variance explained by the QTL. Therefore, the total genetic variance of the population was calculated as $V_{\mathrm{Ga}}=\sum_{j=1}^{p} \beta_{j}^{2} \times 1$, for $j$ th additive (main) effect and $V_{\mathrm{Ge}}=\sum_{j=1}^{p} \beta_{j}^{2} \times \sigma^{2}\left(m l \times m l^{\prime}\right)$ for $j$ th epistatic effect, where $\sigma^{2}\left(m l \times m l^{\prime}\right)$ is the variance of interaction between markers at $l$ and $l^{\prime}$ loci. The total phenotypic variance for the population for each trait was calculated as $V_{\mathrm{P}}=\sum_{i=1}^{n}\left(Y_{i}-\bar{Y}\right)^{2} /(n-1)$, where $Y_{i}$ is the mean value of the $i$ th genotype across environments, $\bar{Y}$ is the average of all the mean values of all the genotypes for a trait, and $n$ is the number of genotypes. The proportion of the phenotypic variance that is explained by the $j$ th QTL was calculated as $H=V_{\mathrm{Ga}} / V_{\mathrm{P}}$ for additive (main) effect and $H=V_{\mathrm{Ge}} / V_{\mathrm{P}}$ for epistatic effect. To declare an estimated effect as "significant," each estimated effect was converted into a $t$ test statistic, $t_{j}=\left|\beta_{j}\right| / S_{\beta_{j}}$, and then further converted in a LOD score using $\operatorname{LOD}_{j}=t_{j}^{2} / 4$.61. All effects with the LOD scores larger than a critical value were declared as significant. The critical value was calculated by a permutation analysis (Churchill and Doerge 1994). The $(1-\alpha) \times 100$ th percentile of the distribution of the LOD scores of the reshuffled sample was a good approximation of the true critical value, where $\alpha$ is a controlled experimental type I error (Xu and Jia 2007).

\section{Results}

We used the eBayes option of PROC QTL (Hu and Xu 2009) to analyze the phenotypic data for different traits (see "Materials and methods"). The eBayes option of PROC QTL implements the empirical Bayes method of Xu 
(2007). The computer program can be downloaded from http://www.statgen.ucr.edu. The critical $t$ values were calculated for all additive (main) and epistatic effects and most stringent values for all the phenotypic traits fell in the $3.8-4.0$ range at an experimental type I error of $\alpha=0.007$, but we chose a maximum critical value of 4.0 to avoid any false detection of QTL (Table 1).

\section{Root traits}

All root characters measured showed significant QTL effects on the short arm of chromosome 1 of rye and a total of 15 QTL effects were found. Six of these were additive and nine showed epistatic interactions (Table 1). Of the nine epistatic interactions, five were inter-genomic interactions between wheat and rye alleles and the rest were intra-genomic interactions. The highest single additive effect explained $57 \%$ of the phenotypic variation for NR; the same effect explained $56 \%$ of the total phenotypic variation for TRL (Table 1). This QTL is tightly linked to marker Pm8, a powdery mildew resistance locus. The highest intra-genomic epistatic effect explained $31 \%$ of the phenotypic variance for DRW with a LOD score of 7.61 (Table 1; Fig. 1e). It was detected between two adjacent regions marked on the map by loci $\operatorname{Pm} 8$ (13) and Gli-1, Glu-3 (14) (Figs. 1e, 2a, b). The highest inter-genomic epistatic effect was detected for NR. This inter-genomic interaction involved Pm8 (13) and Xucr_2 (2) (Fig. 2a, c) and explained $26 \%$ of the phenotypic variation with LOD score of 4.69 (Table 1; Fig. 1a).

Shoot traits

With the exception of shoot biomass, no significant QTL was detected for any shoot trait measured, and no significant QTL effect was detected for the R/S. Two significant intra-genomic epistatic effects were detected for SB. The first one, Xucr_4(5) and Sr31(10) loci explained $42 \%$ $(\mathrm{LOD}=7.34)$ and the second one, Xucr_8(12) and Gli-1, Glu-3(14) explained $16 \%(\mathrm{LOD}=6.80)$ of the phenotypic variation (data not shown).

\section{Mean comparisons}

To check the effect of the presence of 1RS in wheat, the mean of "Pavon 76" was compared with all the recombinant lines with 1RS segments including Pavon 1RS.1BL across the environments. Further, means of different recombinants were compared relative to the position of $1 \mathrm{RS}$ segments in recombinants to validate the mapping of root QTL located in the present study. Pavon 1RS.1BL which contains the entire 1RS arm showed higher means for root traits compared to Pavon 76. The recombinants had, in general, higher means for root traits than Pavon 76 (Table 2). In both groups, 1B+lines and T-lines, the overall means for root traits were higher than that of Pavon
Table 1 Significant genetic effects of different QTL linked with different root traits, namely, number of roots greater than $30 \mathrm{~cm}$ (NR > 30), longest root length (LRL), total root length of roots greater than $30 \mathrm{~cm}$ (TRL), shallow root weight (SRW), deep root weight (DRW), and total root weight (TRW)

\begin{tabular}{|c|c|c|c|c|c|c|}
\hline Root trait & Marker I & Marker II & Genetics effect & $\begin{array}{l}\text { Proportion } \\
\text { of phenotypic variance }\end{array}$ & LOD score & Type of effect \\
\hline \multirow[t]{4}{*}{$\mathrm{NR}>30 \mathrm{~cm}$} & 9 & - & 0.64 & 0.34 & $4.86^{*}$ & Additive \\
\hline & 2 & 13 & 0.79 & 0.26 & $4.69 *$ & Epistatic \\
\hline & 13 & - & 0.83 & 0.57 & $3.90 *$ & Additive \\
\hline & 10 & - & 0.75 & 0.47 & $3.60 *$ & Additive \\
\hline \multirow[t]{2}{*}{ LRL } & 12 & 14 & 2.29 & 0.18 & $5.68 *$ & Epistatic \\
\hline & 5 & - & 2.74 & 0.52 & $3.36^{\mathrm{a}}$ & Additive \\
\hline \multirow[t]{3}{*}{ TRL } & 13 & - & 58.14 & 0.56 & $5.36^{*}$ & Additive \\
\hline & 2 & 13 & 52.30 & 0.24 & $5.03 *$ & Epistatic \\
\hline & 10 & - & 47.52 & 0.4 & $4.04 *$ & Additive \\
\hline \multirow[t]{2}{*}{ SRW } & 4 & 5 & 71.47 & 0.15 & $5.57 *$ & Epistatic \\
\hline & 13 & 14 & 55.55 & 0.18 & $3.53^{*}$ & Epistatic \\
\hline \multirow[t]{2}{*}{ DRW } & 13 & 14 & 32.81 & 0.31 & $7.61 *$ & Epistatic \\
\hline & 4 & 5 & 26.79 & 0.11 & $4.67 *$ & Epistatic \\
\hline \multirow[t]{2}{*}{ TRW } & 4 & 5 & 96.72 & 0.14 & $5.42 *$ & Epistatic \\
\hline & 13 & 14 & 89.77 & 0.23 & $4.88 *$ & Epistatic \\
\hline
\end{tabular}

* Significant LOD scores $(P=0.007)$

a Close to LOD threshold value 
Fig. 1 Three-dimensional graphic representation of QTL effects for six different root traits of the 1RS-1BS recombinant bread wheat population. a Number of roots greater than $30 \mathrm{~cm}(\mathrm{NR}>30)$, b longest root length (LRL), c total root length of roots greater than $30 \mathrm{~cm}($ TRL > 30), d shallow root weight (SRW), e deep root weight (DRW), and f dry root biomass (TRW). The main (additive) effects are on the diagonals and the epistatic effects are on the left triangle of the $3 \mathrm{D}$ plots (the graphical scales for LOD scores in individual graphs are different, as generated by the software program)
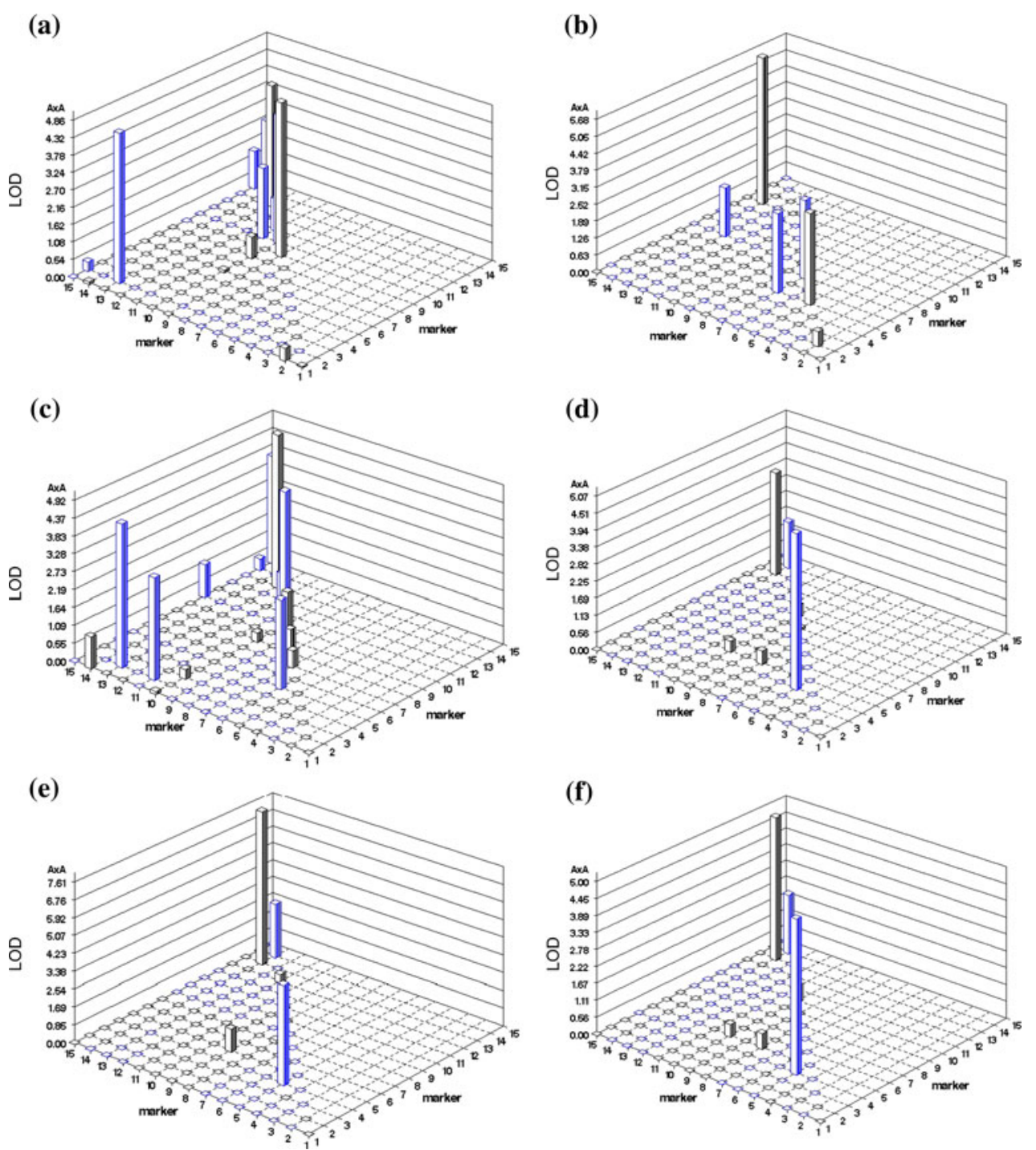

76 , but means of $1 \mathrm{~B}+$ lines were higher than those of T-lines (Table 2).

\section{Discussion}

Preliminary studies (Ehdaie and Waines 2006; Sharma et al. 2009) indicated the distal region of $1 \mathrm{RS}$ covering ca. $15 \%$ of its genetic map, in the genetic background of bread wheat Pavon 76, had a significant effect on root traits. Since root traits are quantitative in nature and controlled by polygenic inheritance (Ehdaie et al. 2001), it was expected to find several QTL associated with the root traits studied.

\section{Advantage of E-Bayesian statistics}

There are many statistical approaches to identify and estimate QTL effects such as linear regression model, interval mapping, maximum-likelihood method, and
Bayesian statistics. Also, there are a number of software, such as MAPMAKER/QTL and QTL Cartographer to map QTL for different phenotypic traits in plants (Price and Tomos 1997; Spielmeyer et al. 2007) as well as in animals (Carlborg et al. 2005; Yi et al. 2006). The E-BAYES method used in this study was chosen for its advantages over other commonly available software and methods. E-BAYES outperformed all other Bayesian methods, stochastic search variable selection (SSVS), penalized likelihood (PENAL), and least absolute shrinkage selection operator (LASSO), in terms of minimizing the error variance. It made shrinkage very selective by providing optimal estimates of variance components, with unshrinking of large effects while small effects are shrunk to zero $(\mathrm{Xu}$ 2007). There were reports of an empirical Bayes method developed by other groups (George and Foster 2000; Yuan and Lin 2005) but they did not shrink each regression coefficient by its own prior and also they were not as easy to understand as E-BAYES developed by $\mathrm{Xu}$ (2007). In 
(a)

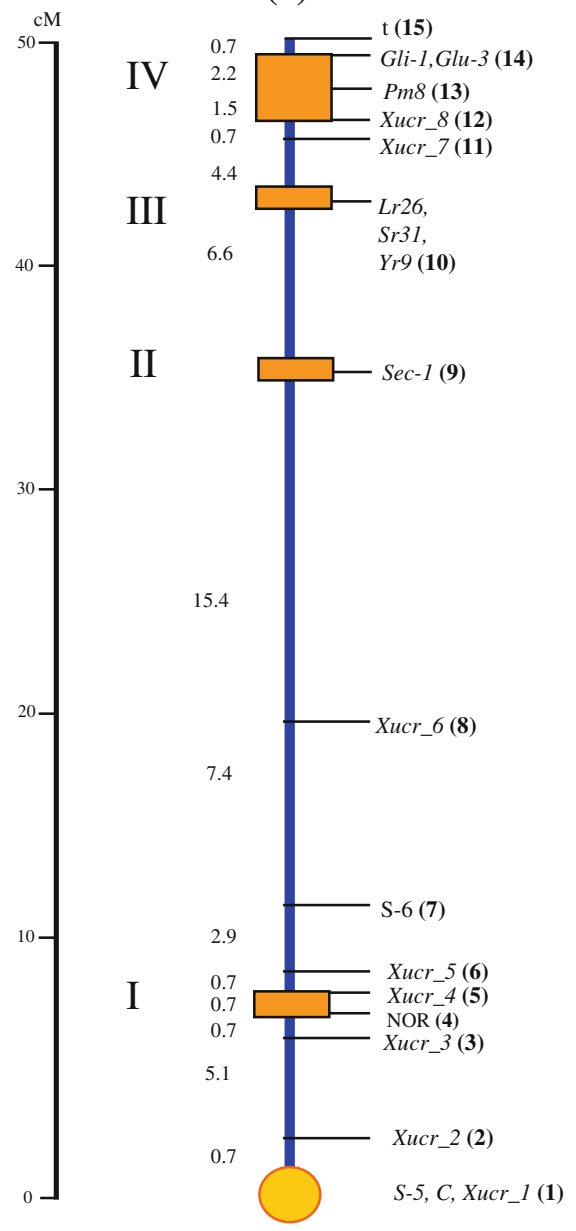

1RS-1BS map
QTL Regions

Additive effect

Fig. 2 Diagrammatic representations of QTL for root traits on the consensus genetic map of 1RS-1BS recombinant lines. a Values on the left side of the 1RS-1BS map are genetic distances and right side of the map show different markers. Values in the parentheses are the number of markers used in this study as reference starting at 1 from the proximal side of the 1RS-1BS map to 15 at the distal end. I, II, III, and IV are the main regions comprising most of the QTL effects for root traits, b location of different QTL effects on 1RS-1BS map,

this study, E-Bayesian approach was applied for the first time in wheat to dissect QTL for root traits.

Chromosome arm-specific mapping population

The mapping population was novel and different from conventional mapping populations. It is analogous to (b)

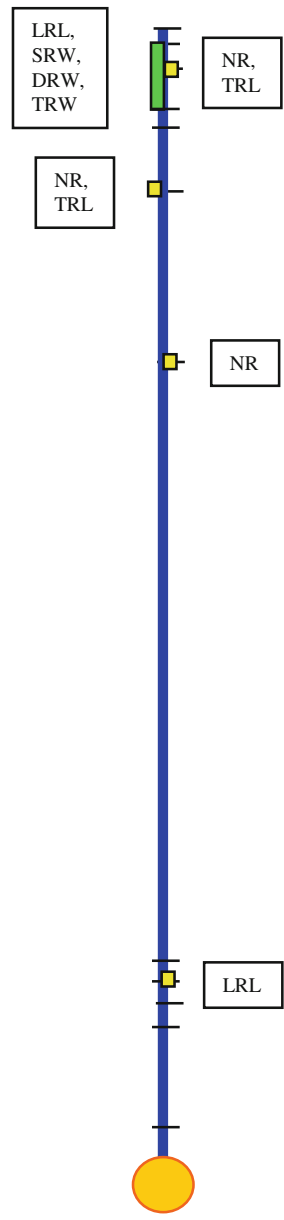

\section{Additive \\ and \\ Intra-genomic epistatic effects}

Intra-genomic epistatic effect (c)

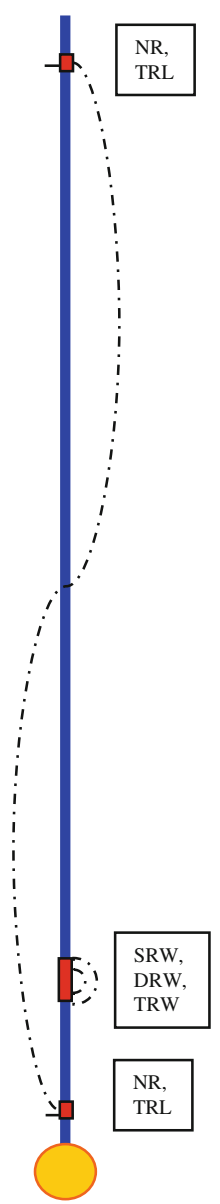

Inter-genomic epistatic effects

yellow rectangles are the additive effects, green rectangles are intragenomic epistatic effects (between rye-rye or wheat-wheat loci), and c location of inter-genomic epistatic interaction (between rye and wheat loci), red rectangles connected by curved dashed line. Boxes with borders show different root traits, $N R$ number of roots greater than $30 \mathrm{~cm}, L R L$ longest root length, $T R L$ total root length of roots greater than $30 \mathrm{~cm}, S R W$ shallow root weight, $D R W$ deep root weight, $T R W$ total root weight

genome-wide introgression lines (ILs) (Eshed and Zamir 1994; Li et al. 2005) where two lines differ for a genomic segment in an otherwise homogeneous background, with the distinction here that the segments in question involved two different genomes (homoeologous recombinants between wheat and rye). In a recent study (Keurentjes et al. 2007), a comparison between mapping power of the RIL 


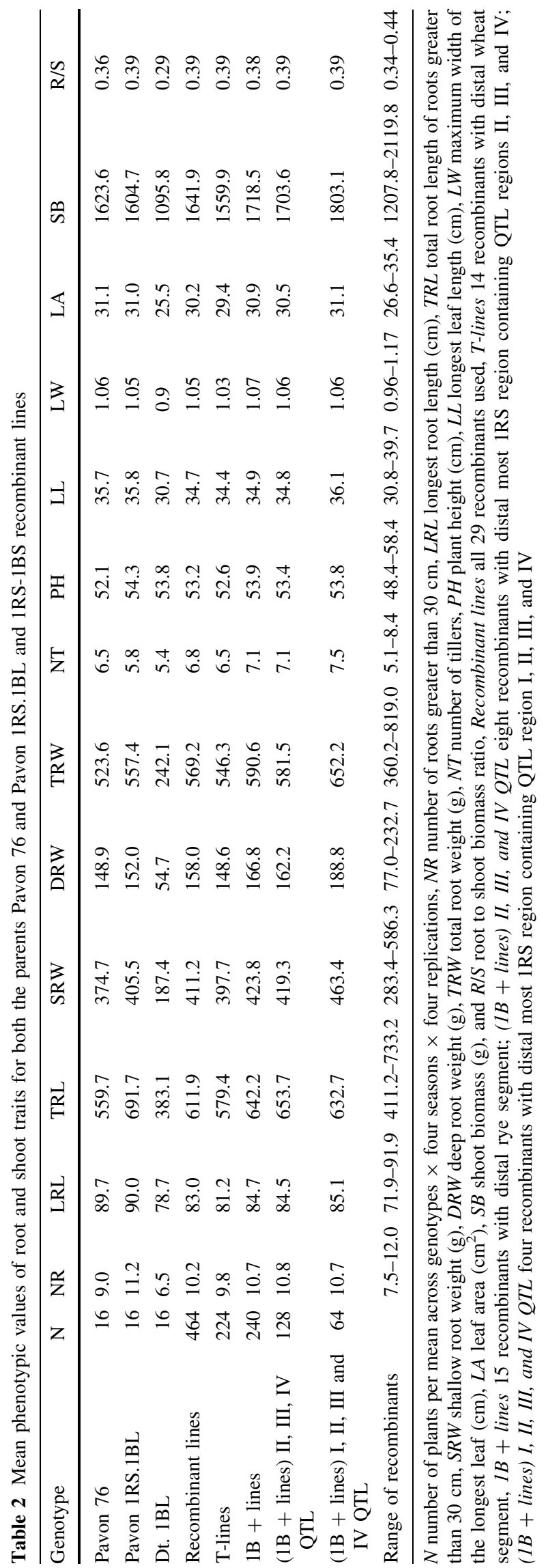

(recombinant inbred line) and NIL populations revealed that population size of RIL is more important than that of replication number where, due to the Beavis effect $(\mathrm{Xu}$ $2003 \mathrm{~b}$ ), the explained variances are overestimated in smaller populations. On the other hand, mapping power of a NIL population rests more on the replication number than population size (Keurentjes et al. 2007). The lower localization resolution of NILs can be increased by generating chromosome- or region-specific mapping populations. The population in this study was a set of single chromosome arm recombinant lines. In such sets, the two parents differ by a single chromosome arm, the remaining chromosome arm pairs being as identical as ca. 10 backcrosses can achieve. This population has statistical advantage in detecting QTL with relatively small phenotypic effects in fewer progeny than by analyzing a large segregating population (Nadeau et al. 2000; Belknap 2003). It is due to the absence of phenotypic noise of segregating unlinked QTL in usual mapping populations with heterogeneous background. It was proved statistically that a $F_{2}$ intercross required a relative increase of at least $37 \%$ more progeny size than a chromosome-specific population to detect single QTL on a chromosome, in the absence of other QTL effects with substantial effect (Singer et al. 2004). This increase would be much larger in the presence of other QTL that contribute to the genetic variance (Singer et al. 2004). Thus, an undetectable effect in a large segregating population can be converted to a detectible effect even in fewer progeny in a chromosomespecific population (Nadeau et al. 2000; Singer et al. 2004). Nadeau et al. (2000) also proved this concept by showing that a population of 28 mice specific for a single chromosome was sufficient for convincing detection of a specific trait locus which could be only weakly detected in an intercross progeny of 300 individuals. This study went a step further using a chromosome arm-specific population. Here, the parent lines differed by the presence/absence of the $1 \mathrm{RS} .1 \mathrm{BL}$ wheat-rye translocation. A set of $1 \mathrm{RS}-1 \mathrm{BS}$ recombinant chromosomes was generated (Lukaszewski 2000), each one originating from a different crossover event and therefore differing from all other recombinants in the proportions of rye and wheat chromatin present. This near-isogenic line approach in essence eliminates the question of population size as a factor in QTL detection. If a QTL is present on the studied arm, it will manifest itself in any properly conducted experiment. The number of lines/recombinants used only affects mapping resolution, that is, the size of the segment to which a trait can be assigned. Thus, 29 lines used in this study, which were selected from a total of 68 recombinants each originating from a single independent crossover, have a maximum resolution of ca. $0.7 \mathrm{cM}$, a feat not frequently achieved in mapping populations. 
Dissection of QTL effects for root traits

We examined a total of 13 phenotypic traits including seven shoot traits and six root traits. A total of 15 QTL effects for root traits were detected using the E-BAYES method. Further partitioning of the root QTL showed six additive effects and nine epistatic effects. Two different types of epistatic effects were recognized that we termed intra-genomic and inter-genomic epistatic effects. Intragenomic epistatic effect resulted from interaction between the alleles of two different loci of either rye or wheat. Intergenomic epistatic effect involved interaction between different alleles of two loci of different genomes, such as one from wheat and one from rye. Pumphrey et al. (2009) reported allelic interactions between different genomes of bread wheat in a synthetic hexaploid T. aestivum. The differential root traits among wheat genotypes with 1RS translocation on 1AL, 1BL, and 1DL (Ehdaie et al. 2003) could likely be due to inter- and intra-genomic epistatic interactions.

\section{Pleiotropy}

Here, most of the additive and epistatic effects detected for variation in different root traits shared common QTL regions. Markers Sr31 (10) and Pm8 (13) shared the QTL with additive (main) effects for NR and TRL (Fig. 2b). One of these markers, $P m 8$, showed inter-genomic epistatic interaction with $X u c r \_2$ (2) and this epistatic effect was again common for NR and TRL (Fig. 2c). NR was affected by four QTL involving almost all markers within the distal $15 \%$ of the 1RS-1BS genetic map. Three QTL effects were involved in the expression of TRL and all three shared the same region as for NR. All three characters for root biomass, viz., SRW, DRW, and TRW, showed two epistatic effects each, and both epistatic effects were common. Pairs of loci involved were NOR (4) and Xucr_4 (5) for intergenomic epistatic effect (Fig. 2c) and Pm8 (13) and Gli-1, Glu-3 (14) for the other intra-genomic epistatic effect (Fig. 2b). Pm8 (13) and Gli-1, Glu-3 (14) were the common loci, also, involved in significant gene interactions even for unrelated root traits of length and weight. This repetitive detection of the associations of the same markers with QTL effects for different root traits consolidates the possibility of either tight linkage or pleiotropy.

Epistasis between loci without main effect

In conventional QTL mapping, the focus is on detecting the QTL with main (additive) effects and then applying an epistatic model to examine the epistatic effect between the QTLs with main effects. In nature, there are loci with small genetic effects which sometimes go undetected in a phenotype but their interaction with other similar loci may have a significant effect. It would be a disadvantage not to include them in the genetic model. Here, we proved the superiority of our method in detecting epistatic effects between two pairs of loci and none of those two pairs of loci had main effects as against other methods where epistatic effects were estimated for loci with main effects only (Kao and Zeng 2002; Maccaferri et al. 2008). This has clearly been shown in the intra-genomic epistatic effects between pairs of loci, Xucr_8 (12) and Gli-1, Glu-3 (14) for LRL. Similar intra- and inter-genomic epistatic effects were also explained for SRW, DRW, and TRW. All these three characters for root biomass showed two pairs of loci involved in two different epistatic interactions and none of these loci had detectable additive effect for these characters.

We studied seven shoot characters for the estimation of QTL effects. Interestingly, we did not find any significant main or epistatic effect for any of the shoot characters except SB. One intra-genomic epistatic effect for SB shared the QTL with LRL (data not shown). A recent QTL study on durum wheat for shoot characters found several QTL on other chromosomes than 1B except for plant height (Maccaferri et al. 2008). Similarly, small QTL effects for NT and PH were detected in this study but they fell short of LOD threshold value (data not shown).

\section{Major QTL regions}

Four regions were identified that carry almost all the QTL with both additive and epistatic effects (Fig. 2). In Fig. 2, they have been marked as I, II, III, and IV as we move from the centromere toward the telomere. Region I was involved in five of the six root traits and covered $0.7 \mathrm{cM}$, which is the highest resolution we could obtain. Region II involved only one marker, Sec-1 (9), showing a single additive effect (NR). Region III covered $0.7 \mathrm{cM}$ and was involved in two main QTL effects for NR and TRL along with one epistatic effect for SB. Region IV covered $3.7 \mathrm{cM}$ with three markers. This region indicated the presence of QTL effects for all the root traits and also for SB. Three of four QTL regions were located in the satellite region of chromosome $1 \mathrm{RS}$. Two of them were located in the distal most $10 \%$ of the $1 \mathrm{RS}$ region which is in agreement with previous studies (Ehdaie and Waines 2006; Sharma et al. 2009).

Validation with mean comparisons

In Table 2, mean comparisons of Pavon 76, Pavon 1RS.1BL, and all the recombinant lines also revealed the higher mean values of root traits in the presence of 1RS chromatin. The presence of distal 1RS segments $(1 \mathrm{~B}+$ lines $)$ showed higher mean values for root traits than the presence of proximal 1RS (T-lines). Lower mean 
values of T-lines may be attributed to the missing distal segment with two markers in all T-lines, one of which belonged to QTL IV. As discussed earlier, three of four QTL regions identified were located in the distal $10 \%$ of the 1RS-1BS arm. For further mean comparisons, $1 \mathrm{~B}+$ lines involving QTL regions IV, III, and II were chosen due to their close proximity with each other. As expected, they showed the higher mean values for root traits compared to T-lines, and significantly higher than those of Pavon 76. The comparison of $1 \mathrm{~B}+$ lines with distal 1RS up to region IV showed higher mean values than Pavon 76, Pavon 1RS.1BL, and all the different sets of recombinant lines. These mean comparisons confirmed the detection of QTL using the E-BAYES method. From the above discussed mean comparisons of recombinant lines, it was obvious the distal part of $1 \mathrm{RS}$ is important for root traits in bread wheat. Hence, we propose the rye loci as major contributors in most of the additive effects and intragenomic epistatic interactions detected for root traits.

Root traits in cereals were associated with drought tolerance. An earlier study (Waines et al. 1998) revealed the association of rye $1 \mathrm{RS}, 2 \mathrm{RS}, 5 \mathrm{R}$, and $7 \mathrm{R}$ chromosomes and chromosome arms with drought tolerance. Other recent studies confirmed the involvement of these rye chromosomes in drought tolerance in the bread wheat background (Koszegi et al. 1996; Mohammadi et al. 2003). In cv. Chinese Spring, all seven rye chromosomes appeared to carry genes influencing drought tolerance and evapo-transpiration efficiency (Waines et al. 1998). The methodology used in this article may provide a general method to analyze these other chromosome arms for QTL analysis and study of epistatic effects.

Here, we addressed the nature of QTL effects for root traits in wheat by studying the additive allelic effects and the intra- and inter-genomic epistatic interactions contributed by rye $1 \mathrm{RS}$. This study provided important information on root genetics which can be pivotal for alien introgressions of genes involved in complex traits. This may also be helpful in marker assisted selection by selecting for a desired combination of alleles for root manipulation toward better adaptability and stability to drought stressed environments. The combined use of a chromosome arm-specific mapping population and an EBayesian approach is novel and makes it possible to study genetic interactions with greater sensitivity and precision than can be done in natural or segregating populations. This may also be another valuable approach to understand complex traits in other organisms.

Acknowledgments This research was supported by United States Department of Agriculture, Southwest Consortium on Plant Genetics and Water Resources, New Mexico, USA Project No. 04N09, the California Agricultural Experiment Station and the University of California, Riverside, Botanic Gardens.
Open Access This article is distributed under the terms of the Creative Commons Attribution Noncommercial License which permits any noncommercial use, distribution, and reproduction in any medium, provided the original author(s) and source are credited.

\section{References}

Belknap JK (2003) Chromosome substitution strains: some quantitative considerations for genome scans and fine mapping. Mamm Genome 14:723-732

Böhm W (1979) Methods of studying root systems. Springer-Verlag, New York

Braun HJ, Payne TS, Morgounov AI, Ginkel Mv, Rajaram S (1998) The challenge: one billion tons of wheat by 2020. In: Slinkard $\mathrm{AE}$ (ed) Proceedings of the 9th international wheat genetics symposium. University Extension Press, University of Saskatchewan, Saskatoon, SK, pp 33-40

Carlborg O, Brockmann GA, Haley CS (2005) Simultaneous mapping of epistatic QTL in DU6i $\times$ DBA/2 mice. Mamm Genome 16:481-494

Champoux MC, Wang G, Sarkarung S, Mackill DJ, O'Toole JC, Huang N, McCouch SR (1995) Locating genes associated with root morphology and drought avoidance in rice via linkage to molecular markers. Theor Appl Genet 90:969-981

Churchill GA, Doerge RW (1994) Empirical threshold values for quantitative trait mapping. Genetics 138:963-971

Ehdaie B, Cress CE (1973) Simulation of cyclic single cross selection. Theor Appl Genet 43:374-380

Ehdaie B, Waines JG (2006) Determination of a chromosome segment influencing rooting ability in wheat-rye 1BS-1RS recombinant lines. J Genet Breed 60:71-76

Ehdaie B, Waines JG (2008) Larger root system increases waternitrogen uptake and grain yield in bread wheat. In: Appels $R$ et al (eds) 11th international wheat genetics symposium 2008. Sydney University Press, Brisbane, p 659

Ehdaie B, Barnhart D, Waines JG (2001) Inheritance of root and shoot biomass in a bread wheat cross. J Genet Breed 55:1-10

Ehdaie B, Whitkus RW, Waines JG (2003) Root biomass, water-use efficiency, and performance of wheat-rye translocations of chromosomes 1 and 2 in spring bread wheat 'Pavon'. Crop Sci 43:710-717

Esau K (1965) Plant anatomy, 2nd edn. Wiley, New York

Eshed Y, Zamir D (1994) A genomic library of Lycopersicon pennelli in L. esculentum: a tool for fine mapping of genes. Euphytica 79:175-179

Falconer DS, Mackay TFC (1996) Introduction to quantitative genetics, 4th edn. Longman, Essex

Fisher RA (1918) The correlations between relatives on the supposition of Mendelian inheritance. Trans R Soc Edinb 52:399-433

George EI, Foster DP (2000) Calibration and empirical Bayes variable selection. Biometrika 87:731-747

Gerald JNF, Lehti-Shiu MD, Ingram PA, Deak KI, Biesiada T, Malamy JE (2006) Identification of quantitative trait loci that regulate Arabidopsis root system size and plasticity. Genetics 172:485-498

Goodnight CJ (2000) Quantitative trait loci and gene interaction: the quantitative genetics of metapopulations. Heredity 84:587-598

Hackauf B, Rudd S, Voort JRvd, Miedaner T, Wehling P (2009) Comparative mapping of DNA sequences in rye (Secale cereale L.) in relation to the rice genome. Theor Appl Genet 118:371-384

Hartley HO, Rao JNK (1967) Maximum-likelihood estimation for mixed analysis of variance model. Biometrika 54:93-108 
Holland JB (2001) Epistasis and plant breeding. Plant Breed Rev 21:27-92

$\mathrm{Hu} \mathrm{Z}$, Xu S (2009) PROC QTL—a SAS procedure for mapping quantitative trait loci. Int J Plant Genomics 2009:1-3

Jannink JL (2003) Selection dynamics and limits under additive $\times$ additive epistatic gene action. Crop Sci 43:489-497

Kao C, Zeng Z (2002) Modeling epistasis of quantitative trait loci using Cockerham's model. Genetics 160:1243-1261

Keurentjes JJB, Bentsink L, Alonso-Blanco C, Hanhart CJ, Blankestijn-De Vries H, Effgen S, Vreugdenhil D, Koornneef M (2007) Development of a near-isogenic line population of Arabidopsis thaliana and comparison of mapping power with a recombinant inbred line population. Genetics 175:891-905

Koszegi B, Farshadfar E, Vagujfalvi A, Sutka J (1996) Drought tolerance studies on wheat/rye disomic chromosome addition lines. Acta Agron Hung 44:121-126

Koumproglou R, Wilkes TM, Townson P, Wang XY, Beynon J, Pooni HS, Newbury HJ, Kearsey MJ (2002) STAIRS: a new genetic resource for functional genomic studies of Arabidopsis. Plant J 31:355-364

Lander ES, Botstein D (1989) Mapping Mendelian factors underlying quantitative traits using RFLP linkage maps. Genetics 121:185-199

Law CN, Snape JW, Worland AJ (1987) Aneuploidy in wheat and its uses in genetic analysis. In: Lupton FGH (ed) Wheat breedingits scientific basis. Chapman \& Hall Ltd, London, pp 71-108

Lebreton C, Lazicjancic V, Steed A, Pekic S, Quarrie SA (1995) Identification of QTL for drought responses in maize and their use in testing causal relationships between traits. J Exp Bot 46:853-865

Li ZK, Fu BY, Gao YM, Xu JL, Ali J, Laffite JR, Jiang YZ, Rey JD, Vijaykumar CHM, Maghirang R, Zheng TQ, Zhu LH (2005) Genome-wide introgression lines and their use in genetic and molecular dissection of complex phenotypes in rice (Oryza sativa L.). Plant Mol Biol 59:33-52

Lukaszewski AJ (2000) Manipulation of the 1RS.1BL translocation in wheat by induced homoeologous recombination. Crop Sci 40:216-225

Maccaferri M, Sanguineti MC, Corneti S, Araus Ortega JL, Salem MB, Bort J, DeAmbrogio E, LFGd Moral, Demontis A, ElAhmed A, Maalouf F, Machlab H, Martos V, Moragues M, Motawaj J, Nachit M, Nserallah N, Ouabbou H, Royo C, Slama A, Tuberosa R (2008) Quantitative trait loci for grain yield and adaptation of durum wheat (Triticum durum Desf.) across a wide range of water availability. Genetics 178:489-511

MacMillan K, Emrich K, Piepho HP, Mullins CE, Price AH (2006) Assessing the importance of genotype $\mathrm{x}$ environment interaction for root traits in rice using a mapping population. I. A soil-filled box screen. Theor Appl Genet 113:977-986

Manske GGB, Vlek PLG (2002) Root architecture-wheat as a model plant. In: Waisel Y, Eshel A, Kafkafi U (eds) Plant roots: the hidden half. Marcel Dekker Inc, New York, pp 249-259

McIntosh RA, Yamazaki Y, Devos KM, Dubcovsky J, Rogers WJ, Appels R (2003) Catalogue of gene symbols for wheat. Wheat Inf Serv 97:27-37

Mettin D, Bluthner WD, Schlegel G (1973) Additional evidence on spontaneous $1 \mathrm{~B} / 1 \mathrm{R}$ wheat-rye substitutions and translocations. In: Sears ER, Sears LMS (eds) Proceedings of the fourth international wheat genetics symposium Alien genetic material. University of Missouri, Columbia, MO, pp 179-184

Mohammadi R, Farshadfar E, Aghaee-Sarbarzeh M, Sutka J (2003) Locating QTLs controlling drought tolerance criteria in rye using disomic addition lines. Cereal Res Commun 31:257-264

Nadeau JH, Singer JB, Matin A, Lander ES (2000) Analysing complex genetic traits with chromosome substitution strains. Nat Genet 24:221-225
Ochoa IE, Blair MW, Lynch JP (2006) QTL analysis of adventitious root formation in common bean under contrasting phosphorus availability. Crop Sci 46:1609-1621

Price AH, Tomos AD (1997) Genetic dissection of root growth in rice (Oryza sativa L.). II. Mapping quantitative trait loci using molecular markers. Theor Appl Genet 95:143-152

Pumphrey M, Bai J, Laudencia-Chingcuanco D, Anderson O, Gill BS (2009) Nonadditive expression of homoeologous genes is established upon polyploidization in hexaploid wheat. Genetics 181:1147-1157

Robinson GK (1991) That BLUP is a good thing: the estimation of random effects. Stat Sci 6:15-32

Sears ER (1953) Nullisomic analysis in common wheat. Am Nat $87: 245-252$

Sharma S, Bhat PR, Ehdaie B, Close TJ, Lukaszewski AJ, Waines JG (2009) Integrated genetic map and genetic analysis of a region associated with root traits on the short arm of rye chromosome 1 in bread wheat. Theor Appl Genet 119:783-793

Shen L, Courtois B, McNally KL, Robin S, Li Z (2001) Evaluation of near-isogenic lines of rice introgressed with QTLs for root depth through marker-aided selection. Theor Appl Genet 103:75-83

Singer JB, Hill AE, Burrage L, Olszens KR, Song JH, Justice M, O'Brien WE, Conti DV, Witte JS, Lander ES, Nadeau JH (2004) Genetic dissection of complex traits with chromosome substitution strains of mice. Science 304:445-448

Spielmeyer W, Hyles J, Joaquim P, Azanza F, Bonnett D, Ellis ME, Moore C, Richards RA (2007) A QTL on chromosome 6A in bread wheat (Triticum aestivum) is associated with longer coleoptiles, greater seedling vigour and final plant height. Theor Appl Genet 115:59-66

Villareal RL, Rajaram S, Mujeeb-Kazi A, Toro ED (1991) The effect of chromosome 1B/1R translocation on the yield potential of certain spring wheats (Triticum aestivum L.). Plant Breed 106:77-81

Waines JG, Ehdaie B, Barnhart DR (1998) Location of genes conferring drought tolerance in rye and bread wheat. In: Jaradat AA (ed) Triticeae III. Proceedings of the third international triticeae symposium, Aleppo, Syria. Science Publishers, Enfield, pp 285-292

Weaver JE (1926) Root development of field crops. McGraw-Hill Book Company Inc, New York

Wu R, Ma C, Casella G (2007) Statistical genetics of quantitative traits-linkage, maps, and QTL. Springer, New York

$\mathrm{Xu} \mathrm{S} \mathrm{(2003a)} \mathrm{Estimating} \mathrm{polygenic} \mathrm{effects} \mathrm{using} \mathrm{markers} \mathrm{of} \mathrm{the} \mathrm{entire}$ genome. Genetics 163:789-801

$\mathrm{Xu} \mathrm{S}$ (2003b) Theoretical basis of the Beavis effect. Genetics 165:2259-2268

Xu S (2007) An empirical Bayes method for estimating epistatic effects of quantitative trait loci. Biometrics 63:513-521

Xu S, Jia ZY (2007) Genomewide analysis of epistatic effects for quantitative traits in barley. Genetics 175:1955-1963

Yi NJ, Xu SZ, Allison DB (2003) Bayesian model choice and search strategies for mapping interacting quantitative trait loci. Genetics 165:867-883

Yi NJ, Zinniel DK, Kim KM, Eisen EJ, Bartolucci A, Allison DB, Pomp D (2006) Bayesian analyses of multiple epistatic QTL models for body weight and body composition in mice. Genet Res 87:45-60

Yuan M, Lin Y (2005) Efficient empirical Bayes variable selection and estimation in linear models. J Am Stat Assoc 100:1215-1225

Zeller FJ, Hsam SLK (1984) Broadening the genetic variability of cultivated wheat by utilizing rye chromatin. In: Sakomoto $S$ (ed) 6th international wheat genetics symposium. Plant Germplasm Institute, Koyoto University, Koyoto, pp 161-173

Zhang M, Montooth KL, Wells MT, Clark AG, Zhang DB (2005) Mapping multiple quantitative trait loci by Bayesian classification. Genetics 169:2305-2318 
Zhang KP, Tian JC, Zhao L, Wang SS (2008) Mapping QTLs with epistatic effects and QTL $\times$ environment interactions for plant height using a doubled haploid population in cultivated wheat. J Genet Genomics 35:119-127
Zheng HG, Babu RC, Pathan MS, Ali L, Huang N, Courtois B, Nguyen HT (2000) Quantitative trait loci for root-penetration ability and root thickness in rice: comparison of genetic backgrounds. Genome 43:53-61 